\title{
Application of the Boundary Element Method Using Time Discretization to the Advection-Convection Equation
}

\author{
Mwesigwa $\mathbf{R}^{1 *}$ and Kakuba $\mathbf{G}^{2}$ \\ ${ }^{1}$ Department of Mathematics, Mbarara University of Science and Technology, Mbarara, Uganda \\ ${ }^{2}$ Department of Mathematics, Makerere University, University Rd, Kampala, Uganda
}

\begin{abstract}
The boundary element method is a numerical computational method of solving partial differential equations which have been formulated as integral equations. It can be applied in many areas of engineering and science including fluid mechanics, acoustics, electromagnetics, and fracture mechanics. The method can be seen as a weighted residual method for solving partial differential equations, characterized by choosing an appropriate fundamental solution as a weighting function and by using the generalized Green's formula for complete transfer of one or more partial differential operators on the weighting function. Time discretization approach requires replacing the partial derivative of the equation that involves time with a finite difference approximation, and the resulting equation now has one variable $\mathrm{x}$ with $\mathrm{t}$ becoming a constant. In this paper the advection-diffusion equation has been formulated using time discretization approach of the boundary element method. The fundamental solution of the elliptic operator has been constructed, and test examples provided.
\end{abstract}

Keywords: Boundary element method; Time discretization

\section{Introduction}

The term boundary element method (BEM) denotes any method for the approximate numerical solution of boundary integral equations, a classical tool for the analysis of boundary value problems for partial differential equations [1-3]. More broadly, BEM has been used as a generic term for a variety of numerical methods that use a boundary or boundary-like discretization [4]. These can include the general numerical implementation of boundary integral equations, known as the boundary integral equation method, whether elements are used in the discretization or not; or the method known as the indirect method that distributes singular solutions on the solution boundary; or the method of fundamental solutions in which the fundamental solutions are distributed outside the domain in discrete or continuous fashion with or without integral equation formulation; or even the Trefftz method which distribute non-singular solutions [5]. BEMs are gaining popularity due to their applications in the vast fields of science and technology and it is also being applied for calculating the solution of compressible fluid flow problems [6].

The collocation BEM, galerkin BEM, dual reciprocity BEM, complex variables BEM, and analog equation method, are some of the variants [7]. Their theoretical and mathematical backgrounds are carefully described and a generalised Laplace's equation (and Poisson's equation) is utilised in demonstrating the different approaches involved. The different algorithms of boundary element method for parabolic equation are presented in, that is, the $1^{\text {st }}$ and $2^{\text {nd }}$ scheme of the BEM [8], the BEM using discretization in time, and the BEM using Laplace transform. The one-dimensional hyperbolic equation supplemented by adequate boundary and initial conditions is considered [9]. This equation is solved using the combined variant of the boundary element method and in an analytical way. It is here concluded that the BEM using discretization in time constitutes the effective numerical method of hyperbolic equation solution but it requires a proper choice of time step and number of internal cells [10].

With the increasing importance of numerical techniques for solving boundary value problems, integral equation methods are becoming more and more popular as a starting point for numerically solving boundary value problems for the reasons listed [11], one of which is, the arising large systems of linear equations are typically better conditioned than the direct finite element discretizations of the underlying boundary value problem. Although integral methods were available many decades ago for the application to flow problems of practical interest, a comprehensive study of the formulation and application to flow problems are still being considered more recently, as they are expected to alleviate sensibly the storage and hopefully CPU time [2]. Despite this apparent advantage, requiring less computational effort when volume integrals are transformed into surface integrals, some disadvantages arise, such as higher mathematical complexity in order to get an usable computational formulation; the need for the calculation of singular integrals; dense matrices whose inversion is more time consuming when comparable with the banded matrices in the finite difference and finite element schemes. Iterative solvers have been proved to be beneficial in finding efficient solvers for BEM systems of equations. Iterative solvers perform matrix-vector multiplication in each iteration, which needs $O\left(N^{2}\right)$ operations in the conventional way and consequently, the total number of operation counts for the BEM with iterative solver is reduced from $O\left(N^{3}\right)$ to $O\left(N^{2}\right)$ [10].

In this paper, solution to the advection-diffusion problem using the time discretization approach of the boundary element method has been discussed. At some stage of the method, one is required to construct the fundamental solution to the elliptic operator, and this has been done. Computations that involve the use of the fundamental solution have been presented and two test examples provided.

*Corresponding author: Mwesigwa R, Department of Mathematics, Mbarara University of Science and Technology, Mbarara, Uganda, Tel: 256-4854-21373 E-mail: rmwesigwa@must.ac.ug

Received October 30, 2018; Accepted November 02, 2018; Published November 23, 2018

Citation: Mwesigwa R, Kakuba G (2018) Application of the Boundary Element Method Using Time Discretization to the Advection-Convection Equation. J App Computat Math 7: 426. doi: 10.4172/2168-9679.1000426

Copyright: (c) 2018 Mwesigwa R, et al. This is an open-access article distributed under the terms of the Creative Commons Attribution License, which permits unrestricted use, distribution, and reproduction in any medium, provided the original author and source are credited. 


\section{Problem Formulation}

We want to solve the following one-dimensional advectiondiffusion equation:

$$
d(x) \frac{\partial^{2} u}{\partial x^{2}}(x, t)=\frac{\partial u}{\partial t}(x, t)+c(x) \frac{\partial u}{\partial x}(x, t)
$$

such that $x \in[a, b]$ and $t \in[0, T]$, with initial condition

$$
u(x, 0)=u_{0}(x) \text {, }
$$

and boundary conditions

$$
u(a, t)=g_{0}(t), u(b, t)=g_{1}(t),
$$

where $c(x)$ and $d(x)$ are arbitrary functions, $d(x) \neq 0$, and we assume that $g_{0}$ and $g_{1}$ are smooth functions over the given interval. In this case though, we will take $c$ and $d$ as constants.

Advection-diffusion models are intended to make predictions through solution of the so called advection-diffusion equation, which makes use of probability, time, velocity and the diffusion coefficient with spatial variability, and reflects two transport mechanisms: advective (or convective) transport with the mean flow, and diffusive transport due to concentrations gradients. The models are typically ran over a medium term period, say, days to months and they are generally limited to small spatial scales.

\section{Developing Boundary Integral Equations}

To solve the eqn. (1), the BEM using discretization in time is applied. We divide the time interval $[0, T]$ into $m$ pieces, each of length $\Delta t=T / m$. The corresponding points are denoted $t_{n}$, for $n=0,1, \ldots, m$. The ends of the interval are $t_{0}=0$ and $t_{m}=T$; the interior points are $t_{n}=n \Delta t$ for $n=1,2, \ldots, m-1$. For the time interval $\left[t_{n}, t_{n+1}\right]$ the following approximation of time derivative can be used

$$
\frac{\partial u}{\partial t}\left(x, t_{n}\right)=\frac{u\left(x, t_{n+1}\right)-u\left(x, t_{n}\right)}{\Delta t}
$$

so that we have

$$
d \frac{\partial^{2} u}{\partial x^{2}}\left(x, t_{n+1}\right)-c \frac{\partial u}{\partial x}\left(x, t_{n+1}\right)-\frac{u\left(x, t_{n+1}\right)-u\left(x, t_{n}\right)}{\Delta t}=0
$$

Let $\beta=0.5 c / d, \gamma=1 / d \Delta t$ and write $u^{n}(x)=u\left(x, t_{n}\right)$. eqn. (1) can be At the $\mathrm{n}$-th time step the approximately rewritten as

$$
\frac{\partial^{2} u^{n+1}(x)}{\partial x^{2}}-2 \beta \frac{\partial u^{n+1}(x)}{\partial(x)}-\gamma u^{n+1}(x)+\gamma u^{n}(x)=0
$$

For eqn. (5), the weighted residual criterion is applied to obtain

$$
\int_{a}^{b}\left(\frac{\partial^{2} u^{n+1}(x)}{\partial x^{2}}-2 \beta \frac{\partial u^{n+1}(x)}{\partial(x)}-\gamma u^{n+1}(x)+\gamma u^{n}(x)=0\right) \omega(x, \xi) d x=0
$$

where $\xi \in(a, b)$ is the observation point, $\omega(x, \xi)$ is the fundamental solution.

Integrating by parts the first two components in eqn. (7) one obtains

$$
\int_{a}^{b} \frac{\partial^{2} u^{n+1}(x)}{\partial x^{2}} w(x, \xi) d x=\left[w(x, \xi) \frac{\partial u^{n+1}(x)}{\partial x^{2}}-\frac{\partial w(x, \xi)}{\partial x} u^{n+1}(x)\right]_{a}^{b}+\int_{a}^{b} u^{n+1}(x) \frac{\partial^{2} w}{\partial x^{2}}(x, \xi) d x
$$

and

$$
\int_{a}^{b} \frac{\partial u^{n+1}(x)}{\partial x} w(x, \xi) d x=\left[w(x, \xi) u^{n+1}(x)\right]_{a}^{b}-\int_{a}^{b} u^{n+1}(x) \frac{\partial w}{\partial x}(x, \xi) d x
$$

so that

$$
\begin{aligned}
& \int_{a}^{b}\left(\frac{\partial^{2} u^{n+1}(x)}{\partial x^{2}}-2 \beta \frac{\partial u^{n+1}(x)}{\partial x}-\gamma u^{n+1}(x)+\gamma u^{n}(x)\right) w(x, \xi) d x \\
& \quad=\left[w(x, \xi) \frac{\partial u^{n+1}(x)}{\partial x}-\frac{\partial w(x, \xi)}{d x} u^{n+1}(x)\right]_{a}^{b}+\int_{a}^{b} u^{n+1}(x) \frac{\partial^{2} w}{\partial x^{2}}(x, \xi) d x \\
& \quad-2 \beta\left[w(x, \xi) u^{n+1}(x)\right]_{a}^{b}+2 \beta \int_{a}^{b} u^{n+1}(x) \frac{\partial w}{\partial x}(x, \xi) d x \\
& \quad-\gamma \int_{a}^{b} w(x, \xi) u^{n+1}(x) d x+\gamma \int_{a}^{b} w(x, \xi) u^{n}(x) d x
\end{aligned}
$$

This may be simplified as

$$
\begin{aligned}
& {\left[w(x, \xi) \frac{\partial u^{n+1}(x)}{d x}-\frac{\partial w(x, \xi)}{\partial x} u^{n+1}(x)-2 \beta w(x, \xi) u^{n+1}(x)\right]_{a}^{b}} \\
& +\int_{a}^{b}\left(\frac{\partial^{2} w}{\partial x^{2}}+2 \beta \frac{\partial w}{\partial x}-\gamma w\right) u^{n+1}(x) d x+\gamma \int_{a}^{b} u^{n}(x) w(x, \xi) d x=0 \\
& \omega(b, \xi) q^{n+1}(b)-\omega^{\prime}(b, \xi) u^{n+1}(b)-2 \beta \omega(b, \xi) u^{n+1}(b) \\
& \text { Or }-\omega(a, \xi) q^{n+1}(a)+\omega^{\prime}(a, \xi) u^{n+1}(a)+2 \beta \omega(a, \xi) u^{n+1}(a) \\
& -u^{n+1}(\xi)+P^{n}(\xi)=0 \\
& \text { Where } q^{n+1}(x)=\frac{\partial u^{n+1}(x)}{\partial x} \\
& \text { And } P^{n}(\xi)=\gamma \int_{a}^{b} u^{n}(x) \omega(x, \xi) d x
\end{aligned}
$$

For $\xi \rightarrow a^{+}$, one obtains after rearranging the terms

$$
\begin{aligned}
& u^{n+1}(a)+\omega(a, a) q^{n+1}(a)-\omega(b, a) q^{n+1}(b) \\
& =\omega^{\prime}(a, a) u^{n+1}(a)+2 \beta \omega(a, a) u^{n+1}(a) \\
& -\omega^{\prime}(b, a) u^{n+1}(b)-2 \beta \omega(b, a) u^{n+1}(b)+P^{n}(a)
\end{aligned}
$$

and in a similar way as $\xi \rightarrow b^{-}$one has

$$
\begin{aligned}
& u^{n+1}(b)+\omega(a, b) q^{n+1}(a)-\omega(b, b) q^{n+1}(b) \\
& =\omega^{\prime}(a, b) u^{n+1}(a)+2 \beta \omega(a, b) u^{n+1}(a) \\
& -\omega^{\prime}(b, b) u^{n+1}(b)-2 \beta \omega(b, b) u^{n+1}(b)+P^{n}(b)
\end{aligned}
$$

In eqn. (12) can now be written as

$$
\begin{aligned}
& {\left[\begin{array}{l}
u^{n+1}(a) \\
u^{n+1}(b)
\end{array}\right]+\left[\begin{array}{l}
\omega(a, a)-\omega(b, a) \\
\omega(a, b)-\omega(b, b)
\end{array}\right]\left[\begin{array}{l}
q^{n+1}(a) \\
q^{n+1}(b)
\end{array}\right]} \\
& =\left[\begin{array}{l}
\omega^{\prime}(a, a)+2 \beta \omega(a, a)-\omega^{\prime}(b, a)-2 \beta \omega(b, a) \\
\omega^{\prime}(a, \mathrm{~b})+2 \beta \omega(a, b)-\omega^{\prime}(b, \mathrm{~b})-2 \beta \omega(b, \mathrm{~b})
\end{array}\right]\left[\begin{array}{l}
u^{n+1}(a) \\
u^{n+1}(b)
\end{array}\right]+\left[\begin{array}{l}
P^{n}(a) \\
P^{n}(b)
\end{array}\right]
\end{aligned}
$$

or, with $\overline{\mathbf{u}}=\left[u^{n+1}(a) u^{n+1}(b)\right]^{T}$ and $\overline{\mathbf{q}}=\left[q^{n+1}(a) q^{n+1}(b)\right]^{T}$ in matrix form as

$$
\mathbf{G} \overline{\mathbf{q}}=\mathbf{H} \overline{\mathbf{u}}+\overline{\mathbf{P}}
$$

where the matrices $\mathbf{G}$ and $\mathbf{H}$ are $2 \times 2$ and are defined as

$$
\mathbf{G}=\left[\begin{array}{l}
\omega(a, a)-\omega(b, a) \\
\omega(a, b)-\omega(b, b)
\end{array}\right]
$$

and

$$
\mathbf{H}=\left[\begin{array}{l}
\omega^{\prime}(a, a)+2 \beta \omega(a, a)-1-\omega^{\prime}(b, a)-2 \beta \omega(b, a) \\
\omega^{\prime}(a, b)+2 \beta \omega(a, b)-\omega^{\prime}(b, b)-2 \beta \omega(b, b)-1
\end{array}\right]
$$

This system of equations allows one to find any two of the boundary values $u^{n+1}(a), u^{n+1}(b), q^{n+1}(a)$, or $q^{n+1}(b)$. The right hand side of this system can be evaluated using numerical integration, otherwise the analytical solution to $\mathbf{P}^{n}(\xi)$ is known and shall be used in the section on 
Citation: Mwesigwa R, Kakuba G (2018) Application of the Boundary Element Method Using Time Discretization to the Advection-Convection Equation. J Appl Computat Math 7: 426. doi: 10.4172/2168-9679.1000426

Page 3 of 5

numerical results. Next, the values of $u$ at the internal nodes $\xi \in(a, b)$ are calculated using the formula in eqn. (12), that is

$$
\begin{aligned}
& u^{n+1}(\xi)=\omega(b, \xi) q^{n+1}(b)-\omega^{\prime}(b, \xi) u^{n+1}(b) \\
& -2 \beta \omega(b, \xi) u^{n+1}(b)-\omega(a, \xi) q^{n+1}(a) \\
& +\omega^{\prime}(a, \xi) u^{n+1}(a)+2 \beta \omega(a, \xi) u^{n+1}(a)+P^{n}(\xi)
\end{aligned}
$$

\section{Construction of the Fundamental Solution}

Gnewuch and Saunter [5] noted that in mathematical textbooks and also in engineering software packages usually only integral equations for the prototype operators such as the Laplace operator, the biharmonic operator, the Lame operator, the Stokes operator are discussed and realised numerically. They then develop the relevant integral equations for the general second order elliptic boundary value problems with constant coefficients

$$
L u:=-\operatorname{div}(A \operatorname{grad} u)+2\langle b, \nabla u\rangle+c u,
$$

since in the farfield, i.e., as $|x|$ becomes large, equations with non constant coefficients or nonlinear equations could be linearized. The definition of fundamental solutions for $L$ involves Macdonald functions $K_{v}$ which, for example, are stated [7]. These functions are modified Bessel functions of the second kind and satisfy the differential equation

$$
x^{2} u^{\prime \prime}+x u^{\prime}-\left(x^{2}+v^{2}\right) u=0
$$

for which they are the solution that remains bounded as $x$ tends to infinity on the real line. They can be given by the following integral representations

$$
\begin{aligned}
& K_{v}(x)=\left(\frac{\pi}{2 x}\right)^{\frac{1}{2}} \frac{e^{-x}}{\Gamma\left(v \frac{1}{2}\right)} \int_{0}^{\infty} e^{-t} t^{v-\frac{1}{2}}\left(1+\frac{t}{2 x}\right)^{v-\frac{1}{2}} d t \\
& =\frac{1}{2}\left(\frac{x}{2}\right)^{v} \int_{0}^{\infty} \exp \left(-t-\frac{x^{2}}{4 t}\right) t^{-v-1} d t
\end{aligned}
$$

\section{Theorem 1}

$$
\begin{aligned}
& \text { Let } v=c+\langle b, b\rangle_{A=} 0 . \text { Then } \kappa_{0}: \mathrm{R}^{d} \rightarrow \mathrm{R} \text { defined by } \\
& k_{0}(x)=\left\{\begin{array}{l}
\frac{1}{2 \pi \sqrt{\operatorname{det} A}} e^{\langle b, x\rangle A} \operatorname{In} \frac{1}{|x| A} \text { for } d=2 \\
\frac{1}{(d-2) \omega_{d} \sqrt{\operatorname{det} A}} \frac{e^{\langle b, x\rangle} A}{|x|_{A}^{d-2}} \text { for } d \neq 2
\end{array}\right.
\end{aligned}
$$

where $\omega_{\mathrm{d}}$ is the volume of the unit sphere in $\mathrm{R}^{\mathrm{d}}$, is a fundamental solution of L. For $\nu \neq 0$, there exists $\lambda \in \mathrm{Cl}(-\infty, 0)$ with $\lambda^{2}=v$. A fundamental solution $\kappa_{\lambda}$ is given by

$$
k_{\lambda}(x)=\frac{e^{\langle b, x\rangle A}}{(2 \pi)^{d / 2} \sqrt{\operatorname{det} A}}\left(\frac{|x|_{A}}{\lambda}\right)^{1-\frac{d}{2}} K_{\frac{d}{2}-1}\left(\lambda|x|_{A}\right), x \neq 0
$$

For $d=1$, we obtain

$$
k_{\lambda}(x)=\frac{e^{\langle b, x\rangle A}}{(2 \pi)^{1 / 2} \sqrt{\operatorname{det} A}}\left(\frac{|x|_{A}}{\lambda}\right)^{1 / 2} K_{-1 / 2}\left(\lambda|x|_{A}\right), x \neq 0
$$

Taking this to our problem means that we seek for a fundamental solution $\omega(x, \xi)$ which should fulfill the equation

$$
\frac{\partial^{2} \omega}{\partial x^{2}}(x, \xi)+2 \beta \frac{\partial \omega}{\partial x}(x, \xi)-\gamma \omega(x, \xi)=-\delta(x, \xi)
$$

where $\delta(x, \xi)$ is the Dirac delta function with the property

$$
\int_{-\infty}^{\infty} \delta(x, \xi) u(x) d x=u(\xi)
$$

We can now write the fundamental solution $\omega$ as

$$
\omega(x, \xi)=\left(\frac{|x-\xi|}{2 \pi \lambda}\right)^{1 / 2} e^{\beta(x-\xi)} K_{-1 / 2}(\lambda|x-\xi|), \quad x-\xi \neq 0
$$

where $\lambda^{2=} \gamma+\beta^{2}$ and

$$
K_{-1 / 2(z)}=(2 z)^{-1 / 2} \int_{0}^{\infty} \exp \left(-t-\frac{z^{2}}{4 t}\right) t^{-1 / 2} d t
$$

The solutions to the modified Bessel functions [1] of the first and second kind, may be defined in another way by

$$
\begin{aligned}
& I_{v}(z)=\sum_{k=0}^{\infty} \frac{1}{k ! \Gamma(k+v+1)}\left(\frac{z}{2}\right)^{2 k+v} \\
& K_{v}(z)=\frac{\pi}{2} \frac{I_{-v}(z)-I_{v}(z)}{\sin (v \pi)},
\end{aligned}
$$

respectively, when $v$ is not an integer, and $I_{v}$ and $K_{v}$ are the two linearly independent solutions to the modified Bessel's equation.

Some selected identities are

$$
\begin{aligned}
& I_{-\frac{1}{2}}(z)=\sqrt{\frac{2}{\pi z}} \cosh (z) \text { and } I_{\frac{1}{2}}(z)=\sqrt{\frac{2}{\pi z}} \sinh (z) \\
& I_{v}^{\prime}(z)=\frac{v}{z} I_{v}(x)+I_{v+1}(z)
\end{aligned}
$$

and from these we can write

$$
\begin{aligned}
& K_{-\frac{1}{2}}(z)=\frac{\pi}{2} \frac{I_{\frac{1}{2}}(z)-I_{-\frac{1}{2}}(z)}{\sin \left(-\frac{1}{2} \pi\right)} \\
& =-\frac{\pi}{2} \sqrt{\frac{2}{\pi z}}(\sinh (z)-\cosh (z)) \\
& =\sqrt{\frac{\pi}{2}} z^{-\frac{1}{2}} e^{-z}, z>0
\end{aligned}
$$

Now, the expression for the fundamental solution may be simplified, by substituting for $K_{-1 / 2}(z)$ and taking $z=x-\xi$, as

$$
\begin{aligned}
& \omega(z)=\left(\frac{|z|}{2 \pi \lambda}\right)^{1 / 2} e^{\beta z} \sqrt{\frac{\pi}{2}}(\lambda|z|)^{-\frac{1}{2}} e^{-\lambda|z|} \\
& =\left(\frac{|z|}{2 \lambda \pi} \cdot \frac{\pi}{2} \cdot \frac{1}{\lambda|z|}\right)^{1 / 2} \exp (\beta z-\lambda|z|) \\
& =\frac{1}{2 \lambda} \exp (\beta z-\lambda|z|)
\end{aligned}
$$

It can be verified that

$\omega^{\prime}(z)=\frac{\partial \omega}{\partial z}=\frac{1}{2 \lambda}(\beta-\lambda \operatorname{sgn}(z)) \exp (\beta z-\lambda|z|)$,

and

$$
P^{n}(\xi)=\gamma\left[u^{n}(x) \frac{1}{2 \lambda(\beta-\lambda \operatorname{sgn}(z))} \exp (\beta z-\lambda|z|)\right]_{x=a}^{x=b}
$$

\section{Computations Involving $\omega(x, \xi)$ at Boundary Points}

Consider a region bounded by $0 \leq x \leq 1$ and $0 \leq t \leq 1$, with $c=1.0$ 
Citation: Mwesigwa R, Kakuba G (2018) Application of the Boundary Element Method Using Time Discretization to the Advection-Convection Equation. J Appl Computat Math 7: 426. doi: 10.4172/2168-9679.1000426

Page 4 of 5

and $d=0.01$. On the basis in eqn. (33), we have the following values of $\omega(x, \xi)$

$$
\begin{aligned}
& \omega(0,0)=\frac{1}{2 \lambda} \exp (-\beta(0)-\lambda|0|)=\frac{1}{2 \lambda}, \\
& \omega(1,0)=\frac{1}{2 \lambda} \exp (-\beta(1)-\lambda|1|)=\frac{1}{2 \lambda} \exp (-\beta-\lambda), \\
& \omega(0,1)=\frac{1}{2 \lambda} \exp (-\beta(-1)-\lambda|-1|)=\frac{1}{2 \lambda} \exp (\beta-\lambda), \\
& \omega(1,1)=\frac{1}{2 \lambda} \exp (-\beta(0)-\lambda|0|)=\frac{1}{2 \lambda},
\end{aligned}
$$

and values of $\left(\omega^{\prime}(x, \xi)\right)$ are computed using in eqn. (34) as follows

$$
\begin{aligned}
& \omega^{\prime}\left(0,0^{+}\right)=\frac{1}{2 \lambda}\left(-\beta-\lambda \operatorname{sgn}\left(0^{a}\right)\right) \exp (-\beta(0)-\lambda|0|)=\frac{1}{2 \lambda}(-\beta+\lambda), \\
& \omega^{\prime}(1,0)=\frac{1}{2 \lambda}(-\beta-\lambda \operatorname{sgn}(1)) \exp (-\beta(1)-\lambda|1|)=\frac{1}{2 \lambda}(-\beta-\lambda) \exp (-\beta-\lambda), \\
& \omega^{\prime}(0,1)=\frac{1}{2 \lambda}(-\beta-\lambda \operatorname{sgn}(-1)) \exp (-\beta(-1)-\lambda|-1|)=\frac{1}{2 \lambda}(-\beta+\lambda) \exp (\beta-\lambda), \\
& \omega^{\prime}\left(1,1^{-}\right)=\frac{1}{2 \lambda}\left(-\beta-\lambda \operatorname{sgn}\left(0^{b}\right)\right) \exp (-\beta(0)-\lambda|0|)=\frac{1}{2 \lambda}(-\beta-\lambda),
\end{aligned}
$$

where $\operatorname{sgn}\left(0^{a}\right)$ and $\operatorname{sgn}\left(0^{b}\right)$ stand for the sign of $x-\xi$ as $\xi \rightarrow 0^{+}$and $\xi \rightarrow 1^{-}$, respectively.

Also,

$$
\begin{aligned}
P^{n}(\xi) & =\gamma \int_{0}^{1} u^{n}(x) \omega(x, \xi) d x \\
& =\gamma\left[u^{n}(x) \frac{1}{2 \lambda(-\beta-\lambda \operatorname{sgn}(x-\xi))} \exp (-\beta(x-\xi)-\lambda|x-\xi|)\right]_{0}^{1} \\
& =\gamma\left(u^{n}(1) \frac{1}{2 \lambda(-\beta-\lambda \operatorname{sgn}(1-\xi))} \exp (-\beta(1-\xi)-\lambda|1-\xi|)\right) \\
& -\gamma\left(u^{n}(0) \frac{1}{2 \lambda(-\beta-\lambda \operatorname{sgn}(0-\xi))} \exp (-\beta(0-\xi)-\lambda|0-\xi|)\right)
\end{aligned}
$$

and thus,

$$
\begin{aligned}
& P^{n}(0)=\frac{\gamma}{2 \lambda}\left(u^{n}(1) \frac{1}{-\beta-\lambda} \exp (-\beta-\lambda)-u^{n}(0) \frac{1}{-\beta+\lambda}\right) \\
& P^{n}(1)=\frac{\gamma}{2 \lambda}\left(u^{n}(1) \frac{1}{-\beta-\lambda}-u^{n}(0) \frac{1}{-\beta+\lambda} \exp (\beta-\lambda)\right)
\end{aligned}
$$

\section{Test Examples}

\section{Example 1}

Consider the one-dimensional advection-diffusion in eqn. (1) in a region bounded by $0 \leq x \leq 1$ and $0 \leq t \leq 1$, with $c=1.0$ and $d=0.1$, with exact solution

$$
u(x, t)=\frac{0.025}{\sqrt{0.000625+0.02 t}} \exp \left(-\frac{\left(x+0.5-t^{2}\right)}{0.00125+0.04 t}\right)
$$

Here we started with $\Delta t=0.25$ to obtain $\beta=0.5 c / d=5, \gamma=1 /(d \Delta t)=40$, and $\lambda=\sqrt{\gamma+\beta^{2}}=8.063$. The fundamental solution and its derivative will be computed using the expressions

$$
\begin{aligned}
& \omega(x, \xi)=\frac{1}{2 \lambda} \exp (-\beta(x-\xi)-\lambda|x-\xi|) \\
& \omega^{\prime}(x, \xi)=\frac{1}{2 \lambda}(-\beta-\lambda \operatorname{sgn}(x-\xi)) \exp (-\beta(x-\xi)-\lambda|x-\xi|)
\end{aligned}
$$

Now, taking $\xi \in(0,1)$ and $\Delta x=0.25$, we have these values for $\omega(x, \xi)$ and $\omega^{\prime}(x, \xi)$ at the end points $a=0$ and $b=1$ (Table 1 ).

From these, we obtain two-column matrices $\mathbf{G}_{x}$ and $\mathbf{H}_{x}$ for all values of $\xi$ using the formulae

$$
\begin{aligned}
& \mathbf{G}_{x}=[\omega(a, \xi)-\omega(b, a)] \\
& \mathbf{H}_{x}=\left[\omega^{\prime}(a, \xi)+2 \beta \omega(a, \xi)-\omega^{\prime}(b, \xi)-2 \beta \omega(b, \xi)\right]
\end{aligned}
$$

The values of $u$ at the boundary are computed using the expression for the exact solution (Table 2). using

Now, assuming that $u$ is known at the boundary, $q$ can be obtained

$$
\mathbf{G} \overline{\mathbf{q}}=\mathbf{H} \overline{\mathbf{u}}+\mathbf{P}^{n}
$$

where the symbols are already explained. The values of $\mathbf{G}$ and $\mathbf{H}$ are

$$
\begin{aligned}
& \mathbf{G}=\left[\begin{array}{ll}
0.0620 & -0.0000 \\
0.0029 & -0.0620
\end{array}\right] \\
& \mathbf{H}=\left[\begin{array}{cc}
-0.1899 & 0.0000 \\
0.0379 & -0.8101
\end{array}\right]
\end{aligned}
$$

Table 1 shows exact values for $u$ and computed values $q$, at the boundary. Now we can have the approximations of $u$ for selected values of $x$ and $t$ shown in Table 1. Here, we have used the formula in eqn. (12).

\section{Example 2}

This is a similar problem to one presented in a previous example. However, we apply different initial and boundary conditions, that is Table 3,

$$
u(x, 0)=\sin (x)
$$

for the initial condition, and the boundary conditions will be obtained from the exact solution in Table 4.

\begin{tabular}{|c|c|c|c|c|}
\hline $\boldsymbol{\xi}$ & $\boldsymbol{\omega}(\boldsymbol{a}, \boldsymbol{\xi})$ & $\boldsymbol{\omega}(\boldsymbol{b}, \boldsymbol{\xi})$ & $\boldsymbol{\omega}^{\mathbf{0}}(\boldsymbol{a}, \boldsymbol{\xi})$ & $\boldsymbol{\omega}^{\mathbf{0}}(\boldsymbol{b}, \boldsymbol{\xi})$ \\
\hline 0.00 & 0.0620 & 0.0000 & 0.1899 & -0.0000 \\
\hline 0.25 & 0.0228 & 0.0000 & 0.0883 & -0.0000 \\
\hline 0.50 & 0.0134 & 0.0001 & 0.0411 & -0.0012 \\
\hline 0.75 & 0.0062 & 0.0024 & 0.0191 & -0.0309 \\
\hline 1.00 & 0.0029 & 0.0620 & 0.0089 & -0.8101 \\
\hline
\end{tabular}

Table 1: Values of $\omega(x, \xi)$ at $a$ and $b$.

\begin{tabular}{|c|c|c|c|c|c|}
\hline $\boldsymbol{t}$ & $\mathbf{0 . 0 0}$ & $\mathbf{0 . 2 5}$ & $\mathbf{0 . 5 0}$ & $\mathbf{0 . 7 5}$ & $\mathbf{1 . 0 0}$ \\
\hline$u(0, t)$ & 0.0000 & 0.0013 & 0.2425 & 0.0271 & 0.0004 \\
\hline$u(1, t)$ & 0.0000 & 0.0000 & 0.0000 & 0.0000 & 0.0004 \\
\hline$q(0, t)$ & - & -0.0039 & -0.7595 & -3.2509 & -0.3548 \\
\hline$q(1, t)$ & - & -0.0010 & -0.1830 & -0.0204 & 0.0050 \\
\hline
\end{tabular}

Table 2: Values of $u(x, t)$ at $x=a$ and $x=b$

\begin{tabular}{|c|c|c|c|c|}
\hline$(\boldsymbol{\xi}, \boldsymbol{t})$ & $\Delta \boldsymbol{t}=\mathbf{0 . 2 5}$ & $\Delta \boldsymbol{t}=\mathbf{0 . 1 2 5}$ & $\Delta \boldsymbol{t}=\mathbf{0 . 0 6 2 5}$ & Exact $\boldsymbol{u}$ \\
\hline$(0.25,0.25)$ & 0.0006 & 0.0003 & 0.0002 & 0.0000 \\
\hline$(0.50,0.25)$ & 0.0003 & 0.0001 & 0.0000 & 0.0000 \\
\hline$(0.75,0.25)$ & 0.0001 & 0.0000 & 0.0000 & 0.0000 \\
\hline$(0.25,0.50)$ & 0.1128 & 0.0653 & 0.0282 & 0.0128 \\
\hline$(0.50,0.50)$ & 0.0524 & 0.0176 & 0.0033 & 0.0000 \\
\hline$(0.75,0.50)$ & 0.0240 & 0.0047 & 0.0004 & 0.0000 \\
\hline$(0.25,0.75)$ & 0.0126 & 0.0075 & 0.0032 & 0.2000 \\
\hline$(0.50,0.75)$ & 0.0059 & 0.0020 & 0.0004 & 0.0271 \\
\hline$(0.75,0.75)$ & 0.0027 & 0.0005 & 0.0000 & 0.0001 \\
\hline
\end{tabular}

Table 3: Approximation values of $u(x, t)$ 
Citation: Mwesigwa R, Kakuba G (2018) Application of the Boundary Element Method Using Time Discretization to the Advection-Convection Equation. J Appl Computat Math 7: 426. doi: 10.4172/2168-9679.1000426

Page 5 of 5

\begin{tabular}{|c|c|c|c|c|c|}
\hline $\boldsymbol{t}$ & $\mathbf{0 . 0 0}$ & $\mathbf{0 . 2 5}$ & $\mathbf{0 . 5 0}$ & $\mathbf{0 . 7 5}$ & $\mathbf{1 . 0 0}$ \\
\hline$u(0, t)$ & 0.0000 & -0.2413 & -0.4560 & -0.6324 & -0.7614 \\
\hline$u(1, t)$ & 0.8415 & 0.6648 & 0.4560 & 0.2295 & 0.0000 \\
\hline$q(0, t)$ & - & 0.3789 & 4.5484 & 7.8935 & 10.5920 \\
\hline$q(1, t)$ & - & 11.4427 & 8.3368 & 4.8717 & 1.2772 \\
\hline
\end{tabular}

Table 4: Values of $u(x, t)$ at $x=a$ and $x=b$.

\begin{tabular}{|c|c|c|c|c|}
\hline$(\xi, \boldsymbol{t})$ & $\Delta \boldsymbol{t}=\mathbf{0 . 2 5}$ & $\Delta \boldsymbol{t}=\mathbf{0 . 1 2 5}$ & $\Delta \boldsymbol{t}=\mathbf{0 . 0 6 2 5}$ & Exact $\boldsymbol{u}$ \\
\hline$(0.25,0.25)$ & -0.1122 & -0.0650 & -0.0281 & 0.0000 \\
\hline$(0.50,0.25)$ & -0.0512 & -0.0172 & -0.0032 & 0.2413 \\
\hline$(0.75,0.25)$ & 0.0015 & 0.0100 & 0.0060 & 0.4676 \\
\hline$(0.25,0.50)$ & 0.2121 & -0.1228 & -0.0531 & -0.2353 \\
\hline$(0.50,0.50)$ & -0.0979 & -0.0329 & -0.0061 & 0.0000 \\
\hline$(0.75,0.50)$ & -0.0276 & 0.0012 & 0.0036 & 0.2353 \\
\hline$(0.25,0.75)$ & -0.2941 & -0.1703 & -0.0736 & -0.4448 \\
\hline$(0.50,0.75)$ & -0.1364 & -0.0458 & -0.0086 & -0.2295 \\
\hline$(0.75,0.75)$ & -0.0537 & -0.0072 & 0.0012 & 0.0000 \\
\hline
\end{tabular}

Table 5: Approximation values of $u(x, t)$.

$$
u(x, t)=\exp (-0.1 t) \sin (x-t)
$$

Taking $\Delta t=0.25$ gives the following values of $u$ at the boundary and the corresponding $q$ values starting at the next level Table 5 .

\section{Conclusion}

We have used the time discretization approach of the boundary element method to solve the advection-convection equation. The fundamental solution of the resulting elliptic operator has been derived from Macdonald's functions, and later used in the method. Two test examples were used to show that the method gives good results for small values of $\Delta t$. Solutions for various increments of time have

been compared, that is, $\Delta t=0.25,0.125,0.0625$ and results showed that smaller values of $\Delta t$ give better approximations of the solution.

\section{References}

1. Abramowitz M, Stegun IA (1964) Handbook of Mathematical Functions. Applied Mathematics Series, 55.

2. Camacho RGR, Barbosa JR (2005) The boundary element method applied to incompressible viscous fluid flow. J Braz Soc Mech Sci Eng 27: 456-462.

3. Chen G, Zhou J (2010) Boundary element methods with applications to nonlinear problems. Springer Science \& Business Media, 7.

4. Cheng AHD, Cheng DT (2005) Heritage and early history of the boundary element method. Eng Anal Bound Elem 29: 268-302.

5. Gnewuch M, Sauter SA (1999) Boundary integral equations for second order elliptic boundary value problems.

6. Lupa M, Ladyga EL (2008) Application of the boundary element method using discretization in time for numerical solution of hyperbolic equation. Scientific Research of the Institute of Mathematics and Computer Science 7: 83-92.

7. Macdonald IG (1998) Symmetric functions and Hall polynomials. Oxford university press.

8. Majchrzak E, Ladyga E, Mendakiewicz J, Piasecka Belkhayat A (2004) Different variants of the boundary element method for parabolic equations. Scientific Research of the Institute of Mathematics and Computer Science 3: 127-132.

9. Muhammad G, Shah NA, Mushtaq M (2009) Merits and demerits of boundary element methods for incompressible fluid flow problems. J Am Sci 5: 57-61.

10. Shen L, Liu YJ (2007) An adaptive fast multipole boundary element method for three dimensional acoustic wave problems based on the Burton-Miller formulation. Comput Mech 40: 461-472.

11. Yu KH, Kadarman AH, Djojodihardjo $H$ (2010) Development and implementation of some BEM variants: A critical review. Eng Anal Bound Elem 34: 884-899. 